\title{
Doenças relacionadas ao saneamento ambiental inadequado e indicadores de saneamento
}

Nas últimas décadas, presenciou-se um rápido crescimento das cidades em tamanho, população e densidade, concentrando os problemas e desafiando a sociedade e aqueles que a administram. Atualmente, o Brasil ainda se encontra em posição de atraso no cenário internacional de saneamento, o que implica em prejuízos à qualidade de vida da população e à economia do país. O objetivo deste trabalho foi avaliar o comportamento das internações e óbitos ocasionados por doenças relacionadas ao saneamento ambiental inadequado (DRSAI) e os indicadores de saneamento em 29 municípios do Rio Grande do Sul, no período de 2000 a 2010. Foram utilizados dados do cadastro do Sistema de Informações Hospitalares do SUS e dos Censos de 2000 e 2010. Foi verificado que o número de internações teve declínio no intervalo de anos estudado, no entanto as internações em decorrência de Dorsais não apresentaram o mesmo comportamento. Em 2000, o total de internações foi de 86.135, caindo para 69.749 em 201. Já as internações por DRSAl, em 2000, totalizaram 1.170 e, em 2010, elevando-se para 1.509. Todos os indicadores de saneamento básico, quando avaliados para a região como um todo, apresentaram crescimento no que diz respeito à cobertura dos serviços no período, no entanto, recomenda-se a análise de cada município separadamente.

\section{Related diseases to inadequate environmental sanitation and sanitation indicators}

\begin{abstract}
In recent decades, cities have grown rapidly in terms of size, population and density, concentrating problems and challenging society and those who manage it. At the presen time, Brazil is still in a position of delay in the international scenario of sanitation, which implies in damages to the quality of life of the population and the economy of the country. The aim of this study was to evaluate the behavior of hospitalizations and deaths caused by diseases related to inadequate environmental sanitation (DRSAl) and sanitation indicators in 29 municipalities of Rio Grande do Sul, from 2000 to 2010 . It was used data from the Hospital Information System of the SUS and the Census of 2000 and 2010. It was verified that the number of hospitalizations declined in the range of years studied, howeve the hospitalizations due to DRSAls did not present the same behavior. In 2000 , the total number of hospitalizations was 86,135 , falling to 69,749 in 2010 . Meanwhile, hospitalizations for DRSAI in 2000 totaled 1,170, and in 2010 it increased to 1,509. All indicators of basic sanitation, when evaluated for the region as totally, showed growth in coverage of services in the period, however, it is recommended the analysis of each municipality separately.
\end{abstract}

Keywords: Public Health; Hospitalization; Sanitation; Public Policies.

Topic: Epidemiologia e Saúde Ambiental

Reviewed anonymously in the process of blind peer.
Received: 02/12/2018

Approved: 26/01/2019
Ana Luiza Bertani Dall'Agnol

Universidade Federal de Pelotas, Brasil http://orcid.org/0000-0002-8620-0390 analuizabda@gmail.com

Larissa Loebens (id)

Universidade Federal de Pelotas, Brasil http://lattes.cnpq.br/2881245126256479 http://orcid.org/0000-0002-4270-4183 laryloebens2012@gmail.com

Carolina Faccio Demarco (iD

Universidade Federal de Pelotas, Brasil http://lattes.cnpq.br/3166438553797256 http://orcid.org/0000-0002-3826-404X carol_demarco@hotmail.com

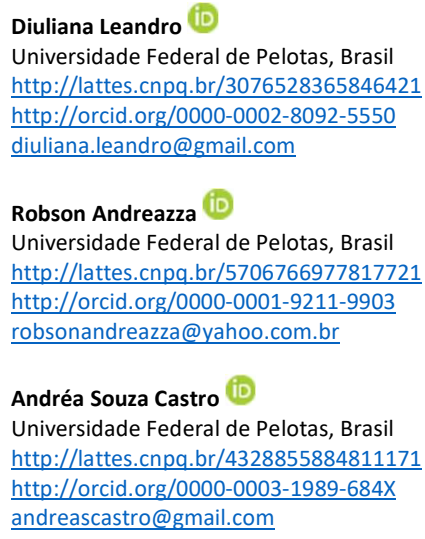
http://lattes.cnpq.br/3076528365846421 http://orcid.org/0000-0002-8092-5550 diuliana.leandro@gmail.com

Robson Andreazza (it)

Universidade Federal de Pelotas, Brasil http://lattes.cnpq.br/5706766977817721 http://orcid.org/0000-0001-9211-9903 robsonandreazza@yahoo.com.br

Andréa Souza Castro (iD)

Universidade Federal de Pelotas, Brasil http://lattes.cnpq.br/4328855884811171 http://orcid.org/0000-0003-1989-684X andreascastro@gmail.com

Maurizio Silveira Quadro (D)

Universidade Federal de Pelotas, Brasil http://lattes.cnpq.br/1749935262841216 http://orcid.org/0000-0001-8236-7479 mausq@hotmail.com

\section{Referencing this:}

SOBRENOME, N. N.; SOBRENOME, N. N.. Doenças relacionadas ao saneamento ambiental inadequado e indicadores de saneamento. Revista Ibero Americana de Ciências Ambientais, v.10, n.1, p.90-98, 2019. DOI: http://doi.org/10.6008/CBPC2179-6858.2019.001.0008 


\section{INTRODUÇÃO}

De acordo com a Lei № 11.445, de 05 de janeiro de 2007, o saneamento básico, no Brasil, compreende o conjunto de serviços, infraestruturas e instalações operacionais de abastecimento de água potável, esgotamento sanitário, limpeza urbana e manejo de resíduos sólidos e drenagem e manejo de águas pluviais (BRASIL, 2007). O saneamento ambiental está intimamente ligado às questões de saúde, portanto, sua falta ou ineficiência contribui para a precariedade dos serviços públicos (FERREIRA et al., 2016).

As Doenças Relacionadas ao Saneamento Ambiental Inadequado (DRSAI) são classificadas pela Fundação Nacional da Saúde - FUNASA e são divididas em cinco grandes grupos: a) doenças de transmissão feco-oral; b) doenças transmitidas por inseto vetor; c) doenças transmitidas através do contato com água; d) doenças relacionadas com a higiene; e e) geo-helmintos e teníases (BRASIL, 2010). Além do saneamento precário, fatores como a falta de políticas públicas e falta de educação sanitária da população favorecem o desenvolvimento e a propagação dessas enfermidades (FERREIRA et al., 2016).

A Organização Pan-Americana da Saúde (OPAS) admitiu, em 2011, que a deficiência no saneamento proporciona um risco à saúde e que, intimamente vinculado à pobreza, atinge mais a população de baixa renda quando associado a outros fatores como subnutrição e falta de higiene. Ainda, segundo a OPAS, a separação entre os campos do planejamento urbano e da saúde corrobora para o fracasso em se reconhecer o ambiente e a saúde das populações mais pobres, sendo assim, o planejamento urbano é uma ferramenta potencialmente poderosa para a melhor e garantir a igualdade social em termos de saúde pública (OPAS, 2011).

Paiva et al. (2018) afirmam que as internações por doenças veiculadas pela água estão associadas às condições de saneamento básico, escolaridade e cobertura por serviços de atenção básica. Já foi também constatada relação entre altas taxas de internações por Doença Diarreica Aguda (DDA) e Doenças Relacionadas ao Saneamento Ambiental Inadequado (DRSAI) com altos índices de pobreza extrema, taxa de analfabetismo e IDH na Amazônia Legal (VIANA et al., 2015).

A situação do saneamento na zona urbana, especialmente em relação ao fornecimento de água potável e a existência rede de esgoto sanitários desempenham uma função fundamental no que diz respeito aos riscos de transmissão de algumas doenças, como por exemplo as diarreias (OLIVEIRA et al., 2015). Quanto maior for a cobertura de serviços adequados de esgotamento sanitário e quanto maior for o PIB per capita, menor é o índice de mortalidade infantil e quanto maior a taxa de analfabetismo, maior é a mortalidade infantil (TEIXEIRA et al., 2011).

Da mesma forma, a leptospirose, enfermidade do grupo de DRSAI, tende a ocorrer em zonas afetadas por problemas de infraestrutura sanitária, como ausência de esgotos, presença de resíduos a céu aberto e locais suscetíveis a inundações. Esses fatores ampliam a vulnerabilidade da população de baixa renda e escolaridade limitada e que residem nessas áreas onde há predisposição para presença do patógeno (GONÇALVES et al., 2016). Neste contexto, o objetivo deste trabalho foi avaliar a ocorrência de internações 
e óbitos por Doenças Relacionadas ao Saneamento Ambiental Inadequado (DRSAI) e os indicadores de saneamento básico em 29 municípios no sul do Estado do Rio Grande do Sul.

\section{MATERIAIS E MÉTODOS}

\section{Área geográfica e população de estudo}

A área geográfica deste trabalho foi composta por 29 municípios do estado do Rio Grande do Sul, pertencentes aos Conselhos Regionais de Desenvolvimento (COREDES) Sul e Campanha, de acordo com a lista da Fundação de Economia e Estatística (FEE) em 2015. A população estudada compreende 1.102.112 habitantes (FEE, 2015).

\section{Base de dados}

A base de dados foi composta por elementos secundários públicos disponíveis no Departamento de Informática do SUS (DATASUS) e por informações do Instituto Brasileiro de Geografia e Estatística (IBGE). A saber: Censo Demográfico 2000; Censo Demográfico 2010; Sistema de Informações Hospitalares do SUS (SIHSUS).

\section{Doenças estudadas}

Foram utilizadas, para o estudo, as Doenças Relacionadas ao Saneamento Ambiental Inadequado DRSAI, indicadas na Tabela 1.

Tabela 1: Doenças Relacionadas ao Saneamento Ambiental Inadequado (DRSAI).

\begin{tabular}{|c|c|c|}
\hline Categoria & Doenças & CID-10 \\
\hline \multirow[t]{2}{*}{1 Doenças de transmissão feco-oral } & Diarreias & A $00 ; A 02-A 04 ; A 06-A 09$ \\
\hline & Febres entéricas & $\mathrm{A} 01$ \\
\hline \multirow[t]{6}{*}{2 Doenças transmitidas por inseto vetor } & Dengue & A90; A91 \\
\hline & Febre amarela & A95 \\
\hline & Leishmanioses & B55 \\
\hline & Filariose linfática & B74 \\
\hline & Malária & B50-B54 \\
\hline & Doença de Chagas & B57 \\
\hline 3 Doenças transmitidas através do contato com a água & $\begin{array}{l}\text { Esquistossomose } \\
\text { Leptospirose }\end{array}$ & $\begin{array}{l}\text { B65 } \\
\text { A27 }\end{array}$ \\
\hline \multirow[t]{3}{*}{4 Doenças relacionadas com a higiene } & Doenças dos olhos & \\
\hline & Tracoma & A71 \\
\hline & Conjuntivites & $\mathrm{H} 10$ \\
\hline \multirow[t]{3}{*}{5 Geo-helmintos e teníases } & Doenças da pele & \\
\hline & Micoses superficiais & B35; B36 \\
\hline & Helmintíases & B68; B69; B71; B76-B83 \\
\hline
\end{tabular}

Fonte: Adaptado de BRASIL (2010).

Para este estudo foram levados em consideração os casos gerais de internação hospitalar e óbitos por local de residência para as doenças selecionadas e para os municípios alvo. Na Tabela 2, estão apresentadas as doenças avaliadas, bem como os respectivos campos selecionados no banco de dados para representá-las. Com exceção de 'doenças dos olhos' e 'doenças da pele', as quais são representadas por Capítulos da CID-10, todos os campos correspondem às opções disponíveis no Sistema de Informações 
Hospitalares do SUS (SIHSUS) em 'Lista Morb CID-10'. O sinal de 'ponto e vírgula' indica que mais de um campo foi selecionado para atender àquela doença.

Tabela 2: Doenças avaliadas e seus respectivos representantes no SIHSUS.

\begin{tabular}{|l|l|}
\hline Doença & Campos correspondentes \\
\hline Diarreias & Diarreia e gastroenterite de origem infeciosa presumível \\
\hline Febres entéricas & Febres tifoide e paratifoide \\
\hline Dengue & Dengue [dengue clássico] \\
\hline Febre amarela & Febre amarela \\
\hline Leishmanioses & Leishmaniose visceral; Leishmaniose cutânea; Leishmaniose cutâneo-musoca; Leishmaniose não especificada* \\
\hline $\begin{array}{l}\text { Filariose } \\
\text { linfática }\end{array}$ & Filariose \\
\hline $\begin{array}{l}\text { Malária } \\
\text { Doença de } \\
\text { Chagas }\end{array}$ & $\begin{array}{l}\text { Malária por Plasmodium falciparum; Malária por Plasmodium vivax; Malária por Plasmodium malariae; Outras } \\
\text { formas de malária conforme exames parasitológicos; Malária não especificada }\end{array}$ \\
\hline $\begin{array}{l}\text { Esquistossomos } \\
\text { e }\end{array}$ & Esquistossomose \\
\hline Leptospirose & Leptospirose icterohemorrágica; Outras formas de lepsospirose; Leptospirose não especificada \\
\hline $\begin{array}{l}\text { Doenças dos } \\
\text { olhos }\end{array}$ & VII. Doenças do olho e anexos \\
\hline Tracoma & Tracoma \\
\hline Conjuntivites & Conjuntivite e outros transtornos da conjuntiva \\
\hline Doenças de pele & XII. Doenças da pele e do tecido subcutâneo \\
\hline $\begin{array}{l}\text { Micoses } \\
\text { superficiais }\end{array}$ & Micoses \\
\hline Helmintíases & Outras helmintíases \\
\hline
\end{tabular}

Fonte: BRASIL (2010) e SIHSUS (2017).

\section{Indicadores de saneamento}

Os indicadores de saneamento foram escolhidos de acordo com os dados fornecidos nos resultados do universo dos censos 2000 e 2010 do IBGE para os municípios alvo do estudo. Assim, os indicadores selecionados foram: abastecimento de água por rede geral; existência de banheiro ou sanitário; rede de esgoto ou pluvial ou fossa séptica; e coleta de resíduos sólidos.

\section{Análise dos dados}

A análise dos dados foi feita por um método evolutivo, organizado em etapas, que permitiram a visualização e determinação dos agravos relacionados ao saneamento ambiental inadequado, de acordo com Teixeira et al. (2014). O método compreendeu, em sequência, as atividades a seguir: Estabelecimento de um padrão para tabulação de dados do DATASUS e IBGE; Coleta dos dados de internações, óbitos e população residente via TabNet Win32 versão 3.0; Coleta dos índices de cobertura de saneamento por meio do Censo 2000 e do Censo 2010 realizados pelo IBGE.

A verificação dos dados compreendeu o período do ano 2000 ao ano 2010 e a justificativa da escolha se deve ao fato de estes serem os anos de realização dos últimos dois censos do IBGE, o que permitiu a comparação e evolução entre os indicadores de saneamento básico para os domicílios particulares permanentes nestes mesmos anos. Para os dados referentes às internações foram realizados os seguintes procedimentos: Coleta de dados de internações totais por local de residência, segundo município, para todos os 29 municípios estudados; Coleta de dados de internações por Doenças Relacionadas ao Saneamento 
Ambiental Inadequado (DRSAI); Somatório de todas as DRSAI encontradas no período; Cálculo do percentual de DRSAl sobre o total de internações; Cálculo da taxa de internações totais para cada 1.000 habitantes (dividindo-se o total de internações pela população residente em cada ano e multiplicando por 1.000); Cálculo da taxa de internações por DRSAI para cada 10.000 habitantes (dividindo-se o total de internações por DRSAI pela população residente em cada ano e multiplicando por 10.000); Em relação aos óbitos, foram seguidos os mesmos passos adotados para as internações.

Para os indicadores de saneamento básico, foram calculados os percentuais de cobertura dos serviços com relação ao total de domicílios para a região estudada: abastecimento de água por rede geral; existência de banheiro ou sanitário; rede de esgoto ou pluvial ou fossa séptica; e coleta de resíduos sólidos. O gráfico foi plotado com o auxílio do programa Microsoft Office Excel 2010.

\section{RESULTADOS}

Do ano 2000 até o ano de 2010 foi constatado um total de 844.920 internações no Sistema Único de Saúde (SUS) de residentes nos municípios alvo deste estudo. Em média ocorreram, aproximadamente, 1.305 internações por ano em função de das Doenças Relacionadas ao Saneamento Ambiental Inadequado - DRSAI, totalizando 14.352 internações em decorrência deste tipo de enfermidade no período.

O comportamento das internações ao longo dos anos demonstrou um declínio (Tabela 3). Pode-se perceber que em 2000, o total de internações foi de 86.135, caindo para 69.749 em 2010, uma redução de 19,0\%. Ao se considerar a população residente da região, em 2000 tinha-se um número de 82,63 internações por cada 1.000 habitantes, caindo para 65,83 em 2010.

Tabela 3: Descrição das internações e óbitos considerando os municípios analisados.

\begin{tabular}{|c|c|c|c|c|c|c|c|c|c|c|}
\hline ANO & INT TOTAL ${ }^{1}$ & INT DRSAI $^{2}$ & $\begin{array}{l}\% \quad \text { INT } \\
\text { TOTAL/ } \\
\text { DRSAI }^{3}\end{array}$ & $\begin{array}{l}\text { INT TOTAL } \\
1.000 \text { hab }^{4}\end{array}$ & $\begin{array}{l}\text { INT DRSAI } \\
10.000 \mathrm{hab}^{5}\end{array}$ & $\begin{array}{l}\text { OB } \\
\text { TOTAL }^{6}\end{array}$ & $\begin{array}{l}\text { OB } \\
\text { DRSAI }^{7}\end{array}$ & $\begin{array}{l}\% \quad \text { OB } \\
\text { TOTAL/D } \\
\text { RSAI }^{8}\end{array}$ & $\begin{array}{l}\text { OB TOTAL } \\
10.000 \\
\mathrm{HAB}^{9}\end{array}$ & $\begin{array}{l}\text { OB DRSAI } \\
10.000 \\
\mathrm{HAB}^{10}\end{array}$ \\
\hline 2000 & 86135 & 1170 & 1,36 & 82,635 & 11,225 & 3327 & 12 & 0,361 & 31,918 & 0,115 \\
\hline 2001 & 87477 & 1158 & 1,32 & 83,141 & 11,006 & 3245 & 9 & 0,277 & 30,842 & 0,086 \\
\hline 2002 & 84088 & 1236 & 1,47 & 79,313 & 11,658 & 3346 & 15 & 0,448 & 31,560 & 0,141 \\
\hline 2003 & 74317 & 948 & 1,28 & 69,556 & 8,873 & 3125 & 14 & 0,448 & 29,248 & 0,131 \\
\hline 2004 & 75776 & 925 & 1,22 & 70,377 & 8,591 & 3611 & 20 & 0,554 & 33,537 & 0,186 \\
\hline 2005 & 73886 & 1486 & 2,01 & 67,451 & 13,566 & 3325 & 23 & 0,692 & 30,354 & 0,210 \\
\hline 2006 & 74663 & 2055 & 2,75 & 67,572 & 18,598 & 3210 & 34 & 1,059 & 29,051 & 0,308 \\
\hline 2007 & 75390 & 1722 & 2,28 & 67,650 & 15,452 & 3471 & 19 & 0,547 & 31,147 & 0,170 \\
\hline 2008 & 71471 & 1064 & 1,49 & 66,101 & 9,841 & 3272 & 16 & 0,489 & 30,262 & 0,148 \\
\hline 2009 & 71968 & 1080 & 1,50 & 66,377 & 9,961 & 3459 & 17 & 0,491 & 31,903 & 0,157 \\
\hline 2010 & 69749 & 1509 & 2,16 & 65,834 & 14,243 & 3651 & 34 & 0,931 & 34,460 & 0,321 \\
\hline
\end{tabular}

${ }_{1}^{1}$ INT TOTAL = Número total de internações no período; ${ }^{2}$ INT DRSAI = Número de internações em decorrência de DRSAI no período; 3\%INT TOTAL/DRSAI = Percentual de internações em decorrência de DRSAI sobre o total de internações no período; ${ }^{4}$ INT TOTAL 1.000 hab. = Taxa de internação para cada 1.000 habitantes no período; ${ }^{5}$ INT DRSAI 10.000 hab. = Taxa de internação por DRSAI para cada 10.000 habitantes no período; ${ }^{6} \mathrm{OB}$ TOTAL: Número total de óbitos no período; ${ }^{7} \mathrm{OB}$ DRSAI: Número de óbitos em decorrência de DRSAI no período; ${ }^{8} \% \mathrm{OB}$ TOTAL/DRSAI: Percentual óbitos em decorrência de DRSAl sobre o total de óbitos no período; ${ }^{9} \mathrm{OB}$ TOTAL 10.000 HAB = Taxa de óbito para cada 10.000 habitantes no período; ${ }^{10} \mathrm{OB}$ DRSAl $10.000 \mathrm{HAB}=$ Taxa de óbitos em decorrência de DRSAI para cada 10.000 habitantes no período.

No entanto, as internações em decorrência de DRSAI não apresentam este comportamento, demonstrando picos de ocorrência, principalmente no ano de 2005 e 2006, decaindo em 2007 e voltando a crescer em 2010. Em 2000 foram 1.170 internações por DRSAI, e em 2010 este número se elevou para 1.509 e o maior valor foi encontrado no ano de 2006, com um total de 2.055 internações. 
O percentual de internações por DRSAI sobre o total de internações, assim como a ocorrência de internações por DRSAI para cada 10.000 habitantes, demonstram o mesmo comportamento que o total de internações por DRSAI. Do total de internações do ano de 2000, as causadas por DRSAI representaram 1,7\%, subindo para 2,2\% em 2010. Considerando a população residente, ocorreram 11,22 internações por DRSAI para cada 10 mil habitantes em 2000 e 14,24 internações por DRSAI para cada 10 mil habitantes em 2010. Em termos monetários, de acordo com o SIHSUS, o custo total das internações por DRSAI para o SUS na região de estudo foi de $R \$ 7.424 .094,67$, o equivalente a 1,3\% do valor gasto com internações hospitalares no período de 2000 a 2010 na mesma região.

Analisando o comportamento dos óbitos ocasionados por DRSAI entre 2000 e 2010 para a região de estudo, verificou-se que os anos de 2006 e 2010 apresentaram os maiores números de casos de óbitos, 34 ocorrências em cada um, o que coincide com os dados apresentados para as internações: 2006 foi o ano de maior número de registros de internações por DRSAI e 2010 o terceiro ano de maior incidência. Em relação aos indicadores de saneamento básico analisados, através da Figura 2, é possível visualizar a cobertura dos serviços de saneamento básico para a região de estudo nos anos 2000 e 2010.

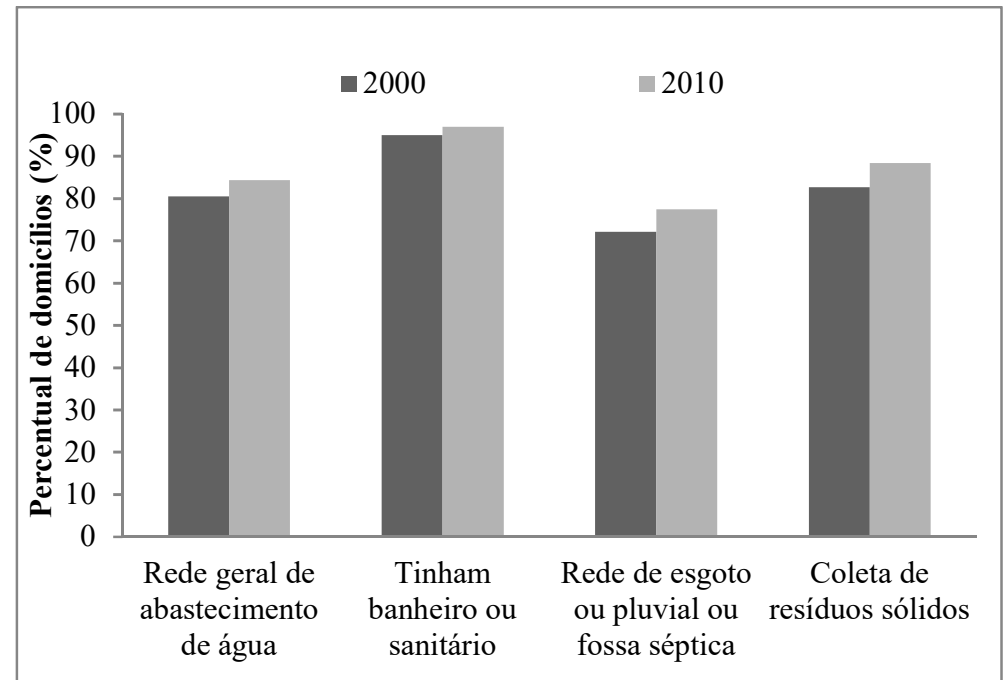

Figura 2: Percentual de domílicios com cobertura dos serviços de saneamento básico para os COREDES Sul e Campanha nos anos 2000 e 2010. Fonte: IBGE (2000) e IBGE (2010).

Os setores que mais apresentaram crescimento no período foram as formas de esgotamento sanitário e a coleta de resíduos sólidos, com incremento de 5,3\% e 5,6\%, alcançando, assim, uma cobertura de $77,4 \%$ e $88,4 \%$, respectivamente. No entanto, em 2010, 3.732 domicílios da região ainda não possuíam banheiro ou sanitário e o resíduo de 41.961 residências tinha outro destino, que não a coleta.

\section{DISCUSSÃO}

O declínio evidenciado nas internações ao longo dos anos pode estar associado ao aumento nos investimentos em saúde no Brasil no período, que passou de 20,3 bilhões em 2000 para 66,7 bilhões em 2010, de acordo com dados do Senado Federal. Já em relação aos picos de ocorrência detectados nas internações em decorrência de DRSAI (em 2000 foram 1.170 internações por DRSAI, e em 2010 este número se elevou para 1509), estes foram diferentes dos resultados apresentados por Teixeira et al. (2014), que 
afirma que o número de internações no Brasil por doenças relacionadas ao saneamento básico inadequado teve uma queda de 5,72\% entre 2001 e 2009.

Nos anos em que se verifica o aumento na incidência de internações por DRSAI, verificou-se que os mesmos coincidem com anos de ocorrência de El Niño, que, de acordo com Britto et al. (2008), é um fenômeno que provoca o aumento anormal da temperatura da superfície do mar no Pacífico Equatorial, podendo afetar os regimes pluviométricos do Rio Grande do Sul, ocasionando, de modo geral, chuvas acima da média. De acordo com o Centro de Previsão do tempo e Estudos Climáticos - CPTEC (2017) ocorreu El Niño moderado em 2002, fraco nos anos de 2005 e 2006 e moderado em 2010, anos onde foram identificados aumentos nas ocorrências de internações.

Em período de chuvas anormais, são maiores as chances de ocorrência de eventos de desastres hidrológicos naturais, que envolvem os processos que resultam em alagamentos, enchentes, inundações graduais e bruscas e movimentos de massa úmida (deslizamentos) (OPS, 2015). Estudos a respeito dos impactos dos desastres naturais sobre a saúde humana afirmam que estes atingem as populações de maneira desigual e de formas distintas, direta e indiretamente, com consequências que variam de curto a longo prazo, dependendo da característica do evento e da vulnerabilidade socioeconômica e ambiental do local onde ocorre (ALDERMAN et al., 2012).

De acordo com a Organização Pan-americana da Saúde (2015), em um curto prazo de tempo, que compreende horas a alguns dias do desastre hidrológico, ocorre a maior parte dos registros de feridos leves e graves e mortalidade. $\mathrm{O}$ segundo momento, entre dias a meses, é caracterizado pelos casos de doenças transmissíveis (leptospirose, influenza, transmissão hídrica ou alimentar), e pela intensificação das não transmissíveis (hipertensão).

Freitas et al. (2012) caracterizaram as consequências ambientais e sobre a saúde provocadas pelas enchentes. Dentre as consequências ambientais se encontram: a) a contaminação da água, do solo e dos alimentos, que comprometem o consumo humano; b) comprometimento dos serviços de saneamento ambiental; c) alteração dos ciclos de vetores, hospedeiros e reservatórios de doenças e nas formas de exposições ambientais humanas. Em relação às consequências sobre a saúde, diversos agravos e doenças podem acometer à população afetada, incluindo quase que a totalidade das enfermidades classificadas como DRSAI.

Conhecer as informações sobre os impactos econômicos das internações hospitalares ocasionados ao SUS é muito pertinente pois pode servir de apoio para o planejamento e tomada de decisão sobre aplicação dos recursos (MARTINS et al., 2017). Quanto aos custos totais das internações por DRSAI para o SUS encontrado na região (1,3\% do valor gasto com internações hospitalares no período de 2000 a 2010), Teixeira et al. (2014) encontrou uma média anual de 3,3\% (sobre o total de internações) em custos com internações por doenças relacionadas ao saneamento ambiental inadequado entre 2001 e 2009. Este recurso poderia ser utilizado de outras formas, como na educação em saúde e sanitária, bem como investido em saneamento, o que contribui para a promoção da saúde preventiva e coletiva (ALVES, 2005). 
Sobre os indicadores de saneamento básico, é positivo o fato de que todos apresentaram incrementos positivos, mas preocupante na medida em que se ressalta que o período avaliado é de uma década e que os serviços ainda estão distantes da universalização. As dificuldades para alcançar a universalização do acesso aos serviços de saneamento ultrapassam as esferas políticas e ideológicas, pois estão também relacionadas às questões institucionais, de financiamento, gestão, tecnologia, participação e controle social (BORJA, 2014).

Como muitos trabalhos já realizados confirmam a associação entre as condições de saneamento e a ocorrência de doenças (BRASIL, 2010; FERREIRA et al., 2016; ANDREAZZI et al., 2007; PAIVA et al., 2017; GUIMARÃES et al., 2013; ERCUMEN et al., 2014), é fundamental que os índices dos indicadores sejam explorados para análise da universalização do acesso a estes serviços e identificação de áreas críticas para o planejamento e adoção de políticas públicas com vistas a melhorar a qualidade de vida da população.

\section{CONCLUSÕES}

De uma maneira geral, a população da região estudada tem adoecido menos, pois o número de internações totais ocorridas teve redução no período de 2000 a 2010, diferentemente do comportamento apresentado pelas internações ocasionadas por DRSAl, que apresentou picos, especialmente nos anos de 2006, 2007 e 2010. O comportamento dos óbitos ocasionados por DRSAl acompanhou o de internações, demonstrando aumento em 2006 e 2010.

No que diz respeito ao saneamento básico, o percentual de cobertura dos serviços na região apresentou crescimento em 2010, quando comparados aos dados de 2000. No entanto, recomenda-se avaliar cada cidade isoladamente para verificar as reais condições e desafios de cada localidade para o alcance da universalização do acesso ao Saneamento Básico.

É importante salientar que as Políticas Nacionais de Saneamento Básico e de Resíduos Sólidos foram estabelecidas nos anos de 2007 e 2010, portanto, no período estudado suas diretrizes ainda estavam em fases de implantação. Nesse sentido, é fundamental realizar estudos após a divulgação das novas Pesquisas Nacionais por Amostras de Domicílios (PNAD) e do Censo 2020, afim de avaliar se a região está melhorando suas condições de saneamento através das políticas públicas recentes. Ainda, recomenda-se que sejam elaboradas pesquisas mais aprofundadas sobre as causas da diferença de comportamento entre as internações totais e as internações por DRSAI.

AGRADECIMENTOS: O presente trabalho foi realizado com apoio da Coordenação de Aperfeiçoamento de Pessoal de Nível Superior - Brasil (CAPES) - Código de Financiamento 001, da Fundação de Amparo à pesquisa do Estado do Rio Grande do Sul - FAPERGS e da Universidade Federal de Pelotas.

\section{REFERÊNCIAS}

ALDERMAN, K.; TURNER, L. R.; TONG, S.. Floods and human health: a systematic review. Environment International, v.47, p.37-47, 2012. DOI:

http://doi.org/10.1016/j.envint.2012.06.003
ALVES, V. S.. Um modelo de educação em saúde para o Programa Saúde da Família: pela integralidade da atenção e reorientação do modelo assistencial. Interface - Comunic, 
Saúde, Educ., v.9, n.16, p.39-52, 2005. DOI: http://doi.org/10.1590/S1414-32832005000100004

ANDREAZZI, M. A. R.; BARCELLOS, C.; HACON, C.. Velhos indicadores para novos problemas: a relação entre saneamento e saúde. Rev Panam Salud Pública, v.22, n.3, p.211-217, 2007.

BRASIL. Fundação Nacional de Saúde. Impactos na saúde e no sistema único de saúde decorrentes de agravos relacionados a um saneamento ambiental inadequado. Brasília: Fundação Nacional de Saúde, 2010.

BRASIL. Lei n.11.445: Estabelece diretrizes nacionais para o saneamento básico; altera as Leis nos 6.766, de 19 de dezembro de 1979, 8.036, de 11 de maio de $1990,8.666$, de 21 de junho de 1993, 8.987, de 13 de fevereiro de 1995; revoga a Lei no 6.528, de 11 de maio de 1978; e dá outras providências. Brasília: DOU, 2007.

BORJA, P. C.. Política pública de saneamento básico: uma análise da recente experiência brasileira. Saúde e Sociedade, São Paulo, v.23, n.2, p.432-447, 2014. DOI: http://doi.org/10.1590/S0104-12902014000200007

BRITTO, F. P.; BARLETTA, R.; MENDONÇA, M.. Variabilidade espacial e temporal da precipitação pluvial do Rio Grande do Sul: influência do fenômeno El Niño Oscilação Sul. Revista Brasileira de Climatologia, v.3, p.1-12, 2008. DOI: http://dx.doi.org/10.5380/abclima.v3i0.25408

CPTEC. Centro de previsão do tempo e estudos climáticos. Ocorrência de El Niño. 2017.

ERCUMEN, A.; GRUBER, J. S.; COLFORD, J. M.. Water distribution system deficiencies and gastrointestinal illness: a systematic review and meta-analysis. Environ Health Perspect, v.122, n.7, p.651-660, 2014. DOI: http://doi.org/10.1289/ehp.1306912

FERREIRA, P. S. F.; MOTTA, P. C.; SOUZA, T.C.; SILVA, T. P.; OLIVEIRA, J. F.; SANTOS, A. S. P.. Avaliação preliminar dos efeitos da ineficiência dos serviços de saneamento na saúde pública brasileira. Revista Internacional de Ciências, Rio de Janeiro, v.6, n.2, p.214-229, 2016. DOI: https://doi.org/10.12957/ric.2016.24809

FREITAS, C. M.; XIMENES, E. F.. Enchentes e saúde pública: uma questão na literatura científica recente das causas, consequências e respostas para prevenção e mitigação. Rev. Ciência \& Saúde Col., v.17, n.6, p.1601-1616, 2012. DOI: http://dx.doi.org/10.1590/S1413-81232012000600023

FEE. Fundação de Economia e Estatística. COREDES, 2015.

GONCALVES, N. V.; ARAUJO, E. N.; SOUZA JUNIOR, A. S.; PEREIRA, W. M. M.; MIRANDA, C. S. C.; CAMPOS, P. S. S.; MATOS, M. W. S.; PALÁCIOS, V. R. C. M. P.. Leptospirosis space-time distribution and risk factors in Belém, Pará, Brazil. Ciênc. saúde coletiva, v.21, n.12, p.3947-3955, 2016. DOI: http://dx.doi.org/10.1590/1413$\underline{812320152112.07022016}$
GUIMARÃES, R. M.; ASMUS, C. L. R. F.; OLIVEIRA JUNIOR, S. A.; MAZOTO, M. L.. Acesso ao saneamento básico e a internação por doença diarreica aguda: um estudo da vulnerabilidade infantil. Revista de Salud Ambiental, v.13, p.22-29, 2013.

IBGE. Instituto Brasileiro de Geografia e Estatística. Censo demográfico 2000: características da população e dos domicílios. Rio de Janeiro, 2000.

IBGE. Instituto Brasileiro de Geografia e Estatística. Censo demográfico 2010: características da população e dos domicílios. Rio de Janeiro, 2011.

MARTINS M. M. F.; TEIXEIRA M. C. P.. Análise dos gastos das internações hospitalares por anemia falciforme no estado da Bahia. Cad. Saúde Colet., v.25, n.1, p.24-30, 2017. DOI: http://doi.org/10.1590/1414-462X201700010209

OLIVEIRA, A. F.; LEITE, I. C.; VALENTE, J. G.. Global burden of diarrheal disease attributable to the water supply and sanitation system in the State of Minas Gerais, Brazil: 2005. Cien Saude Colet, v.20, n.4, p.1027-1036. 2015. DOI: http://dx.doi.org/10.1590/1413-81232015204.00372014

OPAS. Organização Pan-Americana da Saúde. Desastres Naturais e Saúde no Brasil. Brasília: OPAS, Ministério da Saúde, 2015.

OPS. Organización Panamericana de La Salud. Agua y Saneamiento: evidencias para políticas públicas con enfoque en derechos humanos y resultados en salud pública. Washington: OPS, 2011

PAIVA, R. F. P. S.; SOUZA, M. F. P.. Associação entre condições socioeconômicas, sanitárias e de atenção básica e a morbidade hospitalar por doenças de veiculação hídrica no Brasil. Cad. Saúde Pública, v.34, n.1, 2018. DOI: http://doi.org/10.1590/0102-311x00017316

SIHSUS. Sistema de Informações Hospitalares do SUS. Sistema de Informações Hospitalares Descentralizado, Brasília, 2017.

TEIXEIRA, J. C.; GOMES, M. H. R.; SOUZA, J. A.. Análise da associação entre saneamento e saúde nos estados brasileiros: estudo comparativo entre 2001 e 2006. Engenharia Sanitária e Ambiental, v.16 n.2, p.197-204, 2011. DOI: http://doi.org/10.1590/S141341522006000300011

TEIXEIRA, J. C.; OLIVEIRA, G. S.; VIALI, A. M.; MUNIZ, S. S.. Estudo do impacto das deficiências de saneamento básico sobre a saúde pública no Brasil no período de 2001 a 2009. Engenharia Sanitária e Ambiental, v.19, n.1, p.87-96, 2014. DOI: http://doi.org/10.1590/S141341522014000100010

VIANA, R. L.; FREITAS, C. M.; GIATII, L. L.. Saúde ambiental e desenvolvimento na Amazônia legal: indicadores socioeconômicos, ambientais e sanitários, desafios e perspectivas. Saúde Soc., São Paulo, v.25, n.1, p.233-246, 2015. DOI: http://doi.org/10.1590/S0104-12902016140843

A CBPC - Companhia Brasileira de Produção Científica (CNPJ: 11.221.422/0001-03) detém os direitos materiais desta publicação. Os direitos referem-se à publicação do trabalho em qualquer parte do mundo, incluindo os direitos às renovações, expansões e disseminações da contribuição, bem como outros direitos subsidiários. Todos os trabalhos publicados eletronicamente poderão posteriormente ser publicados em coletâneas impressas sob coordenação da Sustenere Publishing, da Companhia Brasileira de Produção Científica e seus parceiros autorizados. Os (as) autores (as) preservam os direitos autorais, mas não têm permissão para a publicação da contribuição em outro meio, impresso ou digital, em português ou em tradução. 